\title{
Beneficial metabolic effects of TREM2 in obesity are uncoupled from its expression on macrophages
}

Omar Sharif ${ }^{1,2,3,4^{*}}$, Julia Stefanie Brunner ${ }^{1,2,3,4,12}$, Ana Korosec ${ }^{1,2,13}$, Rui Martins ${ }^{1,2,14}$, Alexander Jais $^{5,15}$, Berend Snijder ${ }^{2,16}$, Andrea Vogel ${ }^{3,4}$, Michael Caldera ${ }^{6}$, Anastasiya Hladik ${ }^{1,2}$, Karin Lakovits $^{1,2}$, Simona Saluzzo ${ }^{1,2,17}$, Benedikta Boehm ${ }^{1,2}$, Anna-Dorothea Gorki ${ }^{1,2}$, Ildiko Mesteri ${ }^{7}$, Josefine Lindroos-Christensen ${ }^{5,18}$, Katharina Tillmann ${ }^{8}$, Dagmar Stoiber ${ }^{9,10}$, Jörg Menche ${ }^{2,6,11}$, Gernot Schabbauer ${ }^{3,4}$, Martin Bilban ${ }^{5}$, Giulio Superti-Furga ${ }^{2,9}$, Harald Esterbauer ${ }^{5}$ and Sylvia $\mathrm{Knapp}^{1,2^{*}}$

${ }^{1}$ Department of Medicine I, Laboratory of Infection Biology, Medical University of Vienna, Vienna, Austria. ${ }^{2}$ Research Center for Molecular Medicine of the Austrian Academy of Sciences, 1090 Vienna, Austria. ${ }^{3}$ Institute for Vascular Biology, Centre for Physiology and Pharmacology, Medical University Vienna, Vienna, Austria. ${ }^{4}$ Christian Doppler Laboratory for Arginine Metabolism in Rheumatoid Arthritis and Multiple Sclerosis, Vienna, Austria. ${ }^{5}$ Department of Laboratory Medicine, Medical University of Vienna, Vienna, Austria. ${ }^{6}$ Department of Structural and Computational Biology, Max Perutz Labs, University of Vienna, Campus Vienna Biocenter 5, A-1030 Vienna, Austria ${ }^{7}$ Institute of Pathology, Überlingen, Germany. ${ }^{8}$ Center of Biomedical Research, Medical University of Vienna, Vienna, Austria. ${ }^{9}$ Institute of Pharmacology, Center of Physiology and Pharmacology, Medical University of Vienna, Vienna, Austria. ${ }^{10}$ Department of Pharmacology, Physiology and Microbiology, Division Pharmacology, Karl Landsteiner University of Health Sciences, Krems, Austria. ${ }^{11}$ Faculty of Mathematics, University of Vienna, Oskar-Morgenstern-Platz 1, A-1090 Vienna, Austria. ${ }^{12}$ Present address: Cell Biology Program, Sloan Kettering Institute, Memorial Sloan Kettering Cancer Center, New York, NY, USA. 
${ }^{13}$ Present address: Skin, Endothelium Research Division, Department of Dermatology, Medical University of Vienna, Vienna, Austria. ${ }^{14}$ Present address: Instituto Gulbenkian de Ciência, Oeiras, Portugal. ${ }^{15}$ Present address: Helmholtz Institute for Metabolic, Obesity and Vascular Research (HIMAG), Leipzig, Germany. ${ }^{16}$ Present address: Department of Biology, Institute of Molecular Systems Biology, ETH Zurich, Zurich, Switzerland. ${ }^{17}$ Present address: Department of Dermatology, Medical University of Vienna, Vienna Austria. ${ }^{18}$ Present address: Novo Nordisk, Søborg, Denmark.

\section{*Correspondence:}

Omar Sharif: Omar.Sharif@meduniwien.ac.at, Tel: +43-1-40160-31108

Sylvia Knapp: Sylvia.Knapp@ meduniwien.ac.at, Tel: +43-1-40400-5139 


\section{Supplemental Material}

In vivo Insulin Signaling

Mice were fasted for $2 \mathrm{hr}$, anesthetized and injected with insulin $(0.75 \mathrm{U} / \mathrm{kg})$. Livers were removed 5 min post injection and flash frozen in liquid nitrogen prior to tissue homogenization.

\section{$\underline{\text { Indirect Calorimetry }}$}

Body-weight-matched mice were placed for $72 \mathrm{hr}$ in individual metabolic cages with free access to food and water connected to an open-circuit, indirect calorimetry system combined with the determination of spontaneous activity by beam breaking (Oxylet, Panlab-Bioseb). Room temperature was $21-23^{\circ} \mathrm{C}$ and light/dark cycles were of $12 \mathrm{hr}$. The animals were accustomed to the apparatus during the first $24 \mathrm{hr}$, followed by measurements. Oxygen consumption $\left(\mathrm{VO}_{2}\right)$ and carbon dioxide production $\left(\mathrm{VCO}_{2}\right)$ were recorded using a computer-assisted data acquisition program (Metabolism 2.2.01, Panlab Harvard Apparatus). The Respiratory Quotient (RQ) ([RQ] = $\mathrm{VCO}_{2} / \mathrm{VO}_{2}$ ) was measured. Energy expenditure was calculated using the metabolism software and defined as (in kcal/day $\left./ \mathrm{kg}^{0.75}=1.44 \times \mathrm{VO}_{2} \times[3.815+1.232 \times \mathrm{RQ}]\right)$.

\section{Mouse Laboratory Parameters and ELISAs}

Serum alanine transaminase (ALT), Aspartate transaminase (AST), triglycerides, cholesterol and blood cell counts were quantified with tests certified for in vitro diagnostics at the Center of Biomedical Research of the Medical University of Vienna. Adiponectin and MCP-1 levels were quantified using commercially available ELISA kits (R\&D Systems, \# MRP300, DY479). 


\section{$\underline{\text { Tissue Homogenization }}$}

Isolated organs were weighed, 4 times the volume of $0.9 \% \mathrm{NaCl}$ added and homogenized using MagNA Lyser Green Beads and a tissue homogenizer. $50 \mu 1$ of the homogenate was removed for RNA isolation and mixed with $300 \mu \mathrm{l}$ of TRIzol. The remaining homogenate was mixed one to one with Greenberger lysis buffer (containing $300 \mathrm{mM} \mathrm{NaCl}, 30 \mathrm{mM}$ Tris, $2 \mathrm{mM} \mathrm{MgCl}$, $2 \mathrm{mM} \mathrm{CaCl}_{2}$, 1\% Triton X-100, supplemented with 1:50 Protease Inhibitor Cocktail (Sigma-Aldrich \#P8340)), incubated for $20 \mathrm{~min}$ at $4^{\circ} \mathrm{C}$, centrifuged for $10 \mathrm{~min}$ at $3000 \mathrm{rpm}$ and the supernatant containing proteins frozen at $-80^{\circ} \mathrm{C}$ until needed.

\section{$\underline{\text { Stromal Vascular Fraction (SVF) Isolation }}$}

SVF was isolated as previously described (1-3). Epididymal white adipose (eWAT) fat pads or donor eWAT pieces from adipose transplanted mice were removed, cut into small (approximately $2 \mathrm{~mm}$ ) pieces and washed $2 \mathrm{x}$ with DMEM:F12 media (Gibco, \#11320-074) supplemented with 50 $\mu \mathrm{g} / \mathrm{ml}$ gentamycin (PAA Laboratories, \#P11-005) by centrifugation for $8 \mathrm{~min}$ at $1800 \mathrm{rpm}$ at room temperature. Adipose was digested in $10 \mathrm{ml}$ of freshly prepared collagenase II solution $(1 \mathrm{mg} / \mathrm{ml}$ in $1.5 \%$ sterile BSA, in DMEM:F12/Gentamycin) and the fat allowed to digest in a shaking waterbath at $37^{\circ} \mathrm{C}$ for $1 \mathrm{hr}$. Subsequently, the digested adipose-enzyme solution was filtered through a $100 \mu \mathrm{m}$ strainer into $20 \mathrm{ml}$ DMEM:F12 media supplemented with $10 \% \mathrm{FBS}, 50 \mu \mathrm{g} / \mathrm{ml}$ gentamycin (herein referred to as SVF media) and centrifuged at $1800 \mathrm{rpm}$ (8 min, room temperature). Post centrifugation, three phases formed with mature adipocytes floating on top, an intermediate phase and a pellet containing SVF. If the mature adipocytes were used for the preparation of adipocyte conditioned media (ACM), the upper adipocyte layer was transferred into a fresh tube and processed accordingly as described below. The pellet was resuspended in SVF 
media, washed twice and resuspended in $1 \mathrm{ml}$ of erythrocyte lysis buffer (Qiagen, \#79217) for 5 min, after which $12 \mathrm{ml}$ of SVF media was added, cells were centrifuged and the pellet was resuspended in PBS supplemented with 2\% FBS for antibody labelling and flow cytometry.

\section{$\underline{\text { Generation of Adipocyte Conditioned Media }}$}

The mature adipocyte fraction from SVF was washed once with serum-free media and twice with 1x PBS. Subsequently, $2 \mathrm{ml}$ of mature adipocytes were incubated with $5 \mathrm{ml} \mathrm{RPMI} / 1 \%$ Penstrep in a T-25 flask for $24 \mathrm{hrs}$. The medium was then passed through a $100 \mu \mathrm{m}$ strainer, aliquoted and frozen at $-20^{\circ} \mathrm{C}$ until use.

\section{$\underline{\text { Isolation of ATMs }}$}

SVF was isolated from 13 week HFD animals and the BD IMag Cell Separation System was used for isolating ATMs. The positive selection was conducted according to the protocol provided by the manufacturer using biotinylated anti-mouse CD11c antibody (BD Biosciences, \#553800) to select for CD11 ${ }^{+}$ATMs.

\section{Chemotaxis Assays of Bone Marrow Macrophages.}

Bone marrow (BM) macrophages were retrieved from the tibia and the femur of mice and differentiated in RPMI 1640 supplemented with 1\% pen/strep, 10\% FCS and 10\% L929conditioned medium for 7 days as previously described (4). Chemotaxis was assayed using a Transwell Boyden chamber system. Either RPMI or RPMI supplemented with ACM was added to 
12 wells of a 24 well plate. Subsequently, Corning Transwell System filters (pore size $8 \mu \mathrm{m}$, SigmaAldrich, \#CLS3422) were inserted and 5x10 $\mathrm{BM}$ macrophages were added onto each filter and left at $37^{\circ} \mathrm{C}, 5 \% \mathrm{CO}_{2}$ for $6 \mathrm{hrs}$ for migration to occur. Post migration, $500 \mu \mathrm{l}$ of PBS was added to the 12 empty wells and the filters were transferred to the PBS containing wells for washing. Chemotactic stimuli were removed, filters were placed back into empty wells, PBS removed and $500 \mu 1$ of formalin (4\% (v/v) formaldehyde solution) was added. Filters were fixed in formalin for 15 min, stained with $0.2 \%$ crystal violet (Sigma-Aldrich, \#C6158) solution (dissolved in 50\% methanol) for $30 \mathrm{~min}$ and thoroughly washed again with PBS. Before the filters were mounted onto microscope slides, the cells on the upper side of the filters were removed using a cotton stick. Crystal violet fluorescence was imaged in the red channel using an Olympus FSX100 fluorescence microscope (UPLSAPO 10x objective). 3 to 4 random images of each membrane were taken and then loaded into the CellProfiler software (5). Image illumination correction functions were applied to each image and the red channel converted to grayscale images. The image contrast was enhanced using ImageJ. The enhanced images were loaded into the CellProfiler software and the cells were counted using the "IdentifyPrimaryObjects" module setup to use the adaptive two-class Otsu thresholding method with intensity as the parameter to distinguish clumped objects. The values were exported to an Excel spreadsheet and further analyzed using GraphPad Prism.

\section{Adipocyte Cell Size Quantification}

Images of murine WAT for automatic measurement of adipocyte size (area and perimeter) were acquired using an Olympus FSX100 all-in-one microscope system at 10X magnification and image analysis was performed using the CellProfiler software (5). Briefly, the images were loaded onto the software and the analysis pipeline was programmed to convert images to grayscale, using the 
combined weights of 1 for blue, 1.5 for green and 0.5 for red. Binary thresholds of the resulting grayscale images were obtained using a manual threshold value of 0.91 without smoothing. Adipocytes were identified as primary objects using binary thresholds from the previous step and shape as the argument to distinguish clumped objects with a smoothing filter of 40px and local maxima suppression for values shorter than $45 \mathrm{px}$. Cells touching the image borders were excluded from the analysis. Adipocyte shape properties were measured using the cells identified on the previous step and the perimeter and area for each cell was saved to a spreadsheet. The average adipocyte area and perimeter per mouse was then calculated and used for statistical analysis.

\section{Whole-Mount Immunofluorescence}

Epididymal white adipose fat pads from obese mice were removed from the indicated mice and placed in $1 \%$ paraformaldehyde for $10 \mathrm{~min}$ at room temperature. The tissues were washed $3 \mathrm{x}$ with PBS/0.3\% Tween-20 (PBST) and cut into finger nail sized pieces, following which they were blocked in 5\% BSA in PBST for $1 \mathrm{hr}$ and incubated with BODIPYTM 500/510 $\mathrm{C}_{1}, \mathrm{C}_{12}$ (Thermo Fisher Scientific, \#D3823) for $30 \mathrm{~min}$. Subsequently, tissues were washed $2 \mathrm{x}$ for 10 min with PBST, counterstained with DAPI and visualized using a Zeiss LSM 700 Laser Scanning Microscope (Zeiss, Jena, Germany).

\section{$\underline{\text { Isolation of Liver Mononuclear Cells }}$}

Liver mononuclear cells were isolated as previously described $(6,7)$. Mice were sacrificed and the livers were perfused via the portal vein with HBSS, cut into small pieces and digested for $1 \mathrm{hr}$ at $37^{\circ} \mathrm{C}$ in RPMI containing $0.05 \%$ collagenase/dispase (Roche, \#10269638001) and $0.01 \%$ trypsin 
inhibitor (Thermo Fisher Scientific, \#17075029). The liver suspension was pressed through a 40 $\mu \mathrm{m}$ cell strainer, centrifuged at $800 \mathrm{~g}\left(10 \mathrm{~min}, 4^{\circ} \mathrm{C}\right)$ and the cell pellet was resuspended in $10 \mathrm{ml}$ RPMI. Cell suspensions were overlayed onto $15 \mathrm{ml}$ of $33 \%$ (Vol/Vol) Percoll solution (SigmaAldrich, \#P1644) and centrifuged for 30 min (no brake, RT). The supernatant was removed and erythrocytes were lysed by resuspending the pellet in $2 \mathrm{ml}$ of red blood cell lysis buffer for $4 \mathrm{~min}$, after which $8 \mathrm{ml}$ of RPMI was added. Cell suspensions were spun at $800 \mathrm{~g}\left(5-10 \mathrm{~min}, 4^{\circ} \mathrm{C}\right)$, supernatant was removed and the cells were washed twice with $10 \mathrm{ml}$ RPMI. Cells were subsequently resuspended in PBS/2\%FBS and total cell numbers per liver were enumerated using a hemocytometer (Turck chamber) before they were stained for flow cytometry.

Generation of apoptotic thymocytes

For the cleaved caspase 3 control, thymocytes were isolated from C57BL/6 mice and cultured at $6 \times 10^{6}$ in RPMI/10\% FCS. Apoptosis was induced by the addition of $1 \mu \mathrm{M}$ dexamethasone for $16 \mathrm{~h}$.

\section{Flow Cytometry and ATM Sorting}

Isolated SVF or liver mononuclear cells were resuspended in PBS supplemented with 2\% FBS and Fc receptors were blocked with a CD16/CD32 antibody (eBioscience, \#14-0161-82, used at 1:1000) for $10 \mathrm{~min}$ on ice. Thereafter SVF was stained with antibodies to: CD45.2 BV500 (BD Biosciences, \#561487, used at 1:200) or CD45.2 PE (Biolegend, \#103106, used at 1:500) or CD45.2-BV650 (BioLegend, \#109836, used at 1:100), CD45.1 Pacific blue (Biolegend, \#110721, used at 1:100), F4/80 PE-Cy7 (eBioscience, \#25-4801-82, used at 1:360) or F4/80 PerCP Cy5.5 (eBioscience, \#45-4801-82, used at 1:100) or F4/80-FITC (BioLegend. \#123108, used at 1:100), 
CD11b PerCP Cy5.5 (eBioscience, \#45-0112-82 used at 1:100) or CD11b Pacific Blue (Biolegend, \#101223, used at 1:500) or CD11b-BV605 (BioLegend, \#101237, used at 1:500), CD11b-PB (Invitrogen, \#RM2828, used at 1:100), CD11c AF700 (eBioscience, \#56-0114-82, used at 1:80) or CD11c FITC (BD Biosciences, \#553801, used at 1:500), CD206 AF647 (Biolegend, \#141711, used at 1:600), CD3-APC (eBioscience, \#17-0032-82, used at 1:160) and Ly6G FITC (Biolegend, $\# 127605$, used at $1: 10,000)$ for $30 \mathrm{~min}$ at $4^{\circ} \mathrm{C}$.

Isolated liver mononuclear cells were stained with antibodies to: CD45.2 BV500 (BD Biosciences, \#561487, used at 1:100), B220 PerCP Cy5.5 (eBioscience, \# 45-0452-82, used at 1:200), CD3 APC (eBioscience, \#17-0032-82, used at 1:200), Ly6G FITC (Biolegend, \#127605, used at 1:2000), CD11b AF700 (eBioscience, \#56-0112-80, used at 1:500), Ly6C BV605 (Biolegend, \#128035, used at 1:100), F4/80 PE-Cy7 (eBioscience, \#25-4801-82, used at 1:200) for $30 \mathrm{~min}$ at $4^{\circ} \mathrm{C}$. Both SVF cells and liver mononuclear cells were subsequently centrifuged at $1250 \mathrm{rpm}\left(5 \mathrm{~min}, 4^{\circ} \mathrm{C}\right)$ and stained with Fixable viability dye eF780/APC-Cy7 (Thermo Fisher Scientific, \#65-0865-14, used at 1:2000) at room temperature for $15 \mathrm{~min}$ and washed twice with PBS/2\% FBS to allow exclusion of dead cells. Lipid content was assessed by staining the cells with BODIPY 493/503 (Fisher Scientific \#11540326) for 15 to $30 \mathrm{~min}$ at $37^{\circ} \mathrm{C}$.

Blood was stained by directly adding the following antibodies: CD11b AF700 (eBioscience, \#560112-82, used at 1:700), Ly6C BV570 (Biolegend, \#128029, used at 1:100) and Ly6G PE (Biolegend, \#127607, used at 1:1500), CD45.1 APC (Biolegend, \# 110713, used at 1:100) and CD45.2 PE (Biolegend, \#103106, used at 1:100) for $30 \mathrm{~min}$ to $50 \mu 1$ of sample. Cells were lysed by adding $140 \mu \mathrm{L}$ ADG lysis buffer (Andergrub Bioresearch) for $10 \mathrm{~min}$, after which $3.5 \mathrm{ml} \mathrm{H}_{2} \mathrm{O}$ was added for 7 min. Samples were washed twice with PBS/FCS and analyzed by flow cytometry (BD LSR Fortessa). 
For cell sorting, stained ATMs were filtered through a $40 \mu \mathrm{M}$ cell strainer and sorted as live, $\mathrm{CD}^{2} 5^{+} \mathrm{CD}^{-} \mathrm{CD} 11 \mathrm{~b}^{+} \mathrm{F} 4 / 80^{+}$on a FACSAria ${ }^{\mathrm{TM}}$ II flow cytometer (BD).

Control stainings for all flow cytometry included single stainings and unstained cells, which served as compensation controls to ensure that emission spectra under the different fluorescences had minimal overlap. Cells were acquired with LSR Fortessa (BD Biosciences) or with a CytoFLEX S Flow Cytometer (Beckman Coulter) and data further analyzed with FlowJo software (TreeStar). ATMs were identified as live, $\mathrm{CD} 45^{+} \mathrm{CD} 11 \mathrm{~b}^{+} \mathrm{F} 4 / 80^{+}$cells and were further sub-gated for CD11c and CD206. Liver macrophages were identified as CD45+CD3-B220-Ly6G ${ }^{-}{ }^{-} \mathrm{CD} 11 \mathrm{~b}^{+} \mathrm{Ly}^{-} \mathrm{C}^{+} \mathrm{F} 4 / 80^{+}$ cells. Neutrophils were defined as $\mathrm{CD} 11 \mathrm{~b}^{+} \mathrm{F} 4 / 80^{-} \mathrm{Ly}_{6 \mathrm{G}^{+}}$, circulating monocytes as $\mathrm{CD} 11 \mathrm{~b}^{+} \mathrm{F} 4 / 80^{-}$

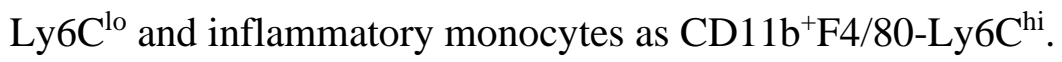

Histology, Immunohistochemistry, Liver Oil Red O and Steatosis Scoring

Organs were fixed in $7.5 \%$ formaldehyde, embedded in paraffin, cut in $2 \mu \mathrm{M}$ slices and stained using hematoxylin and eosin (H\&E). TREM-2 immunohistochemistry on formalin-fixed, paraffinembedded mouse eWAT sections was conducted using the TSA Biosystem kit (Perkin Elmer \#NEL700A001KT). Briefly, sections were de-paraffinised in xylene and rehydrated in graded ethanol followed with blocking of endogenous peroxidase in $3.5 \% \mathrm{H}_{2} \mathrm{O}_{2}$ in PBS for 10 min. Thereafter, sections were subjected to antigen-retrieval using citrate buffer $\mathrm{pH} 6.0$ (Vector laboratories), incubated with TNB blocking reagent for $45 \mathrm{~min}$ and then probed with sheep polyclonal mouse anti-TREM-2 biotinylated antibody (R\&D Systems, \#BAF1729) at a dilution of 1:200, overnight at $4^{\circ} \mathrm{C}$. Subsequently slides were washed with TNT buffer and incubated with SA-HRP according to the manufactures instructions (Perkin Elmer, \#NEL700A001KT). Binding was visualized using the Vectastain ABC kit (Vector laboratories, \#PK-6100) and sections were 
lightly counter stained with haematoxylin (Mayer, Merck), dehydrated, coverslipped and subjected to light microscopy.

Ceramide immunohistochemistry was conducted on xylene de-paraffinised sections, rehydrated in graded ethanol followed with blocking of endogenous peroxidase and alkaline phosphatase in $3.5 \%$ $\mathrm{H}_{2} \mathrm{O}_{2}$ in PBS for 10 min, after which the sections were permbealized with $0.1 \%$ Triton-X in PBS. Thereafter, citrate buffer $\mathrm{pH} 6.0$ (Vector laboratories) was used for antigen-retrieval and sections were incubated with $5 \%$ goat serum for $10 \mathrm{~min}$ and probed with ceramide monoclonal antibody which recognizes C16 and C24 ceramide species (Clone MID 15B4, Enzo Life Sciences, \#ALX804-196-T050) at a dilution of 1:500, overnight at $4^{\circ} \mathrm{C}$. Subsequently slides were washed with PBS, incubated with Biotin-SP-conjugated AffiniPure Fab Fragment goat anti-mouse IgG $(\mathrm{H}+\mathrm{L})$ (Jackson Immuno Research, \# 115-067-003) at a dilution 1:500 for $30 \mathrm{~min}$ at room temperature. Binding was visualized using the Vectastain ABC kit (Vector laboratories, \#PK-6100) and DAB Substrate Kit, Peroxidase (Vector laboratories, \# SK-4100). Sections were stained with haematoxylin (Mayer, Merck), dehydrated, coverslipped and subjected to light microscopy.

TREM-2 and F4/80 double immunohistochemistry was performed on xylene de-paraffinised sections, rehydrated in graded ethanol followed with blocking of endogenous peroxidase in $3.5 \%$ $\mathrm{H}_{2} \mathrm{O}_{2}$ and Levamisole (Fa. Vector, \# SP-5000-18) in PBS for $10 \mathrm{~min}$. Thereafter, sections were subjected to antigen-retrieval using citrate buffer $\mathrm{pH} 6.0$ (Vector laboratories), incubated with 2.5 $\%$ horse serum for 20 min and probed with Rabbit Polyclonal anti-TREM2 Antibody (THP, \#PTG13483-1-AP) at a dilution of 1:200, overnight at $4^{\circ} \mathrm{C}$. Subsequently slides were washed with TBST buffer and incubated with ImmPRESS® HRP Horse Anti-Rabbit IgG Polymer (Vector laboratories, \# MP-7401) for 30 min at room temperature. Binding was visualized using the DAB Substrate Kit, Peroxidase (Vector laboratories, \# SK-4100). Sections were then incubated with 5\% 
donkey serum for 20 min and then probed with Rabbit monoclonal [SP115] against F4/80 (Abcam, \#ab240946) at a dilution 1:100 for $60 \mathrm{~min}$ at room temperature. Subsequently slides were washed with TBST and incubated with Rabbit AP Polymer from the DoubleStain IHC Kit (Abcam, \#ab183285) for 30 min at RT. Binding was visualized using the Permanent Red chromogen from the same kit. Sections were stained with haematoxylin (Mayer, Merck), dehydrated, coverslipped and subjected to light microscopy.

For liver oil Red $\mathrm{O}$ staining, $8 \mu \mathrm{M}$ liver cryosections were stained with oil red $\mathrm{O}(1 \% \mathrm{w} / \mathrm{v}$ isopropanol, diluted 3:2 in PBS) for $15 \mathrm{~min}$ at room temperature and counterstained with hematoxylin according to standard procedures. Liver steatosis was scored on H\&E sections by an experienced pathologist who was blinded for the experimental groups. Macrovesicular steatosis and microvesicular steatosis were both separately scored and defined by whether the vacuoles displaced the nucleus to the side by a single fat globule (macrovesicular) or not due to vast numbers of small fat vesicles (microvesicular) and graded from 0-100\%.

\section{$\underline{\text { Western blotting }}$}

Liver tissue protein content was determined using the Peirce Protein Assay Kit (Thermo Scientific, \#23225). $20 \mu \mathrm{g}$ of proteins were resolved by SDS-PAGE and transferred to PVDF membranes (Biorad). Membranes were blocked with 5\% BSA in PBS containing 0.05\% Tween-20 (PBS-T) and incubated with primary antibodies at $4^{\circ} \mathrm{C}$ overnight at a concentration of 1:1000. The following antibodies were used: Akt Pan (Cell Signaling, \#4691), Phospho-Akt Ser473 (Cell Signaling, \#4060), Vincullin (Cell Signaling, \#4650), Caspase 3 (Cell Signaling, \#9662). Subsequently, membranes were washed 3 times for 10 min with PBST before incubation with anti-rabbit $\operatorname{IgG}$ (Cell Signaling, \#7074, used at 1:1000 in 5\% BSA/PBST). Thereafter, membranes were washed 
again 3x 10 min with PBST and the desired protein was detected using enhanced chemiluminescence.

\section{Quantitative Real Time PCR (qRT-PCR)}

qRT-PCR was performed as described (8). In brief, total RNA was extracted from respective tissues and cells using TRIzol according to the manufacturer's instructions (Ambion, \#15596-018). For RNA purification and cDNA synthesis, equal amounts of RNA (up to $1 \mu \mathrm{g}$ ) were resuspended in $13 \mu \mathrm{H}_{2} \mathrm{O}$ and $1.5 \mu \mathrm{l} 10 \mathrm{x}$ buffer plus $0.5 \mu \mathrm{l}$ DNase I taken from the PerfeCta Dnase I Kit (Quanta Biosciences, \#95150-01K) for DNA digestion. Samples were incubated at $37^{\circ} \mathrm{C}$ for 30 min before 1.5 $\mu \mathrm{l}$ Stop solution was added and incubated thereafter at $65^{\circ} \mathrm{C}$ for another $10 \mathrm{~min}$. Reverse transcription into cDNA was conducted using the iScript cDNA Synthesis Kit according to the manufacturer's instructions (Bio-Rad, \#170-8891). qRT-PCR was performed using the iTaq SYBR Green Supermix with ROX according to the supplier (Bio-Rad, \#72-5853) or the Luna Universal qPCR Master Mix (New England Biolabs \#M3003E). PCR was performed with the StepOnePlus ${ }^{\mathrm{TM}}$ Real-Time PCR System (Applied Biosystems). Post amplification melting curve analysis was performed to check for unspecific products and primer-only controls were included to ensure the absence of primer dimers as well as $\mathrm{H}_{2} \mathrm{O}$ only controls to check for contamination by genomic DNA. For normalization threshold cycles (Ct-values) were normalized to the housekeepers, Hypoxanthine Guanine Phosphoribosyltransferase (Hprt) or Glyceraldehyde 3-phosphate dehydrogenase $\left(\right.$ Gapdh) within each sample to obtain sample-specific $\Delta \mathrm{Ct}$ values $\left(=\mathrm{Ct}_{\text {gene of interest }}\right.$ $\mathrm{Ct}_{\text {housekeeper). }} 2-\Delta \Delta \mathrm{Ct}$ values were calculated to obtain fold expression levels, where $\Delta \Delta \mathrm{Ct}=$ $\left(\Delta \mathrm{Ct}_{\text {treatment }}-\Delta \mathrm{Ct}_{\text {control }}\right)$. Primer sequences are indicated in Supplementary Table 1. 


\section{$\underline{\text { Targeted Metabolomics }}$}

Lipidomics was conducted on $20 \mu \mathrm{l}$ of serum samples or 50-100mg of homogenized adipose tissue from the indicated mice using a targeted metabolomics approach with BIOCRATES Life Sciences AG (Innsbruck, Austria) as previously described $(9,10) .429$ metabolites including 14 amino-acids, 28 hexoses, 40 acylcarnitines, 198 glycerophospholipids (lysophosphatidylcholines (lysoPC), phosphatidylcholines (PC), phosphatidylethanolamines (PE), phosphatidylglycerols (PG), and phosphatidylserines (PS)) and 149 sphingolipids (ceramides (Cer) and sphingomyelins (SM)) were measured. Metabolites were quantified using the in-house validated Partial Lipid Assay and the AbsoluteIDQ ${ }^{\circledR}$ p150 Assay (BIOCRATES Life Sciences AG, Innsbruck, Austria) using tandem mass spectrometry on an AB SCiex triple-quadrupole mass spectrometer operating in positive and negative MRM mode with analysis performed using the MetIDQ software (BIOCRATES Life Sciences AG, Innsbruck, Austria). In a second set of lipidomics samples employing myrocin treatment or adipose tissue samples, metabolites were only quantified using the in-house validated Partial Lipid Assay and for tissue samples resultant lipid pmol were normalized to adipose weight.

The lipidomics were normalized based on the sum of concentrations for all lipid species measured in a single biological replicate. Values were subsequently averaged over biological replicates for HFD conditions (4 per genotype) and $\log _{2}$ transformed against the corresponding average concentrations measured in ND mice (3 per genotype). Significance of pairwise comparisons was calculated using a non-paired two-tailed two-sample $t$-test. Correlations between relative lipid abundance and functional readouts were calculated as Pearson's linear correlation coefficients. For display purposes adjusted Hill-curves were fit to selected relations between relative lipid abundance and functional readouts. 
Further analysis regarding the impact of myriocin was conducted in python 3.7.9. Lipidomic values are normalized between 0 (minimum) and 1 (maximum) for each lipid individually. This type of normalization is particular suitable for the vastly differing concentration ranges between the individual lipids. Values smaller than the limit of detection $(<\mathrm{LOD})$ were set to zero for further calculations. When calculating group averages, lipids were first averaged before the normalization step was performed. When calculating difference between groups of experiments, $\log _{2}$ transformed fold changes were calculated. 


\section{Supplemental Figures}

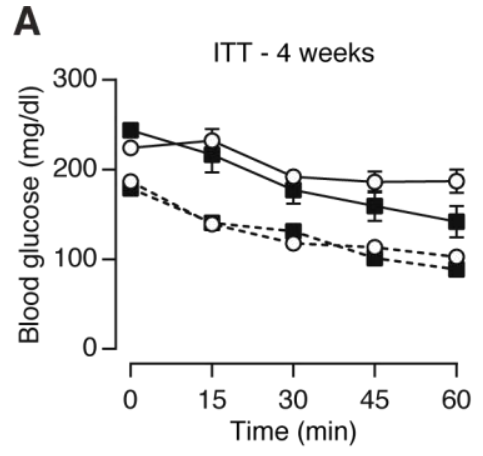

B

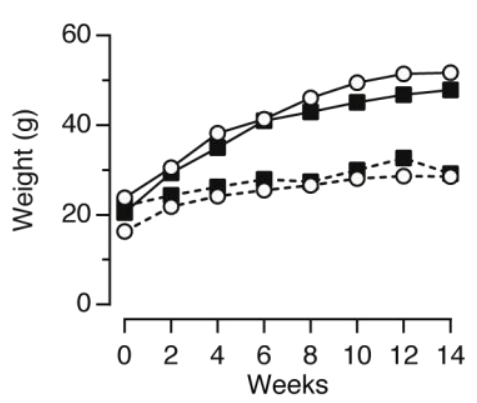

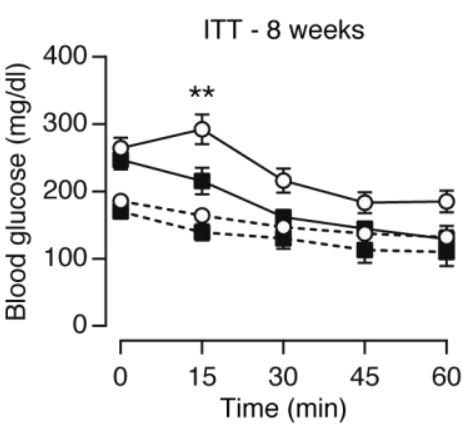

C

ITT - 26 weeks

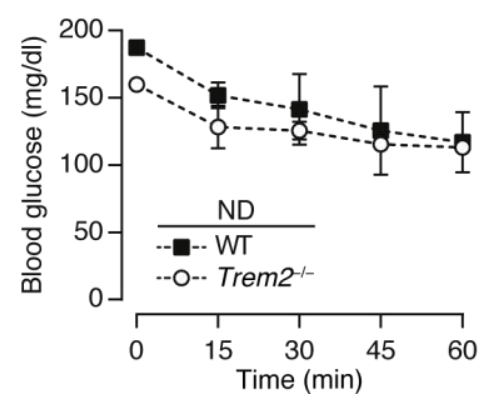

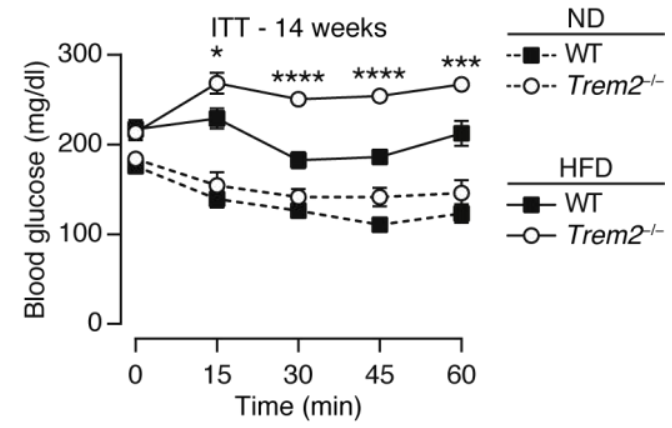

D

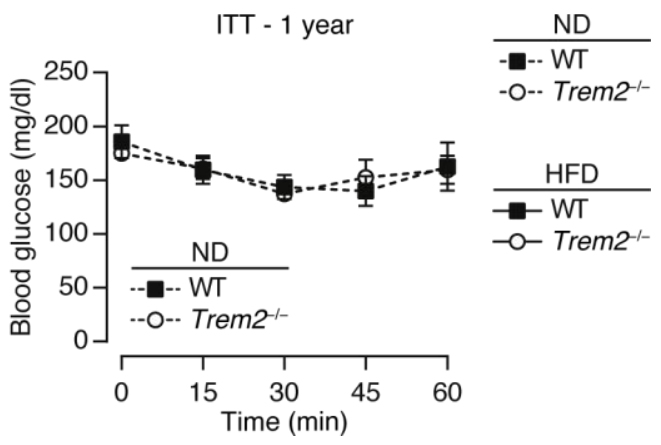

Supplemental Figure. 1 TREM2 only influences metabolic health following metabolic stress.

(A) Insulin tolerance test post normal chow $\operatorname{diet}(\mathrm{ND})$ or HFD at the indicated times, $\mathrm{n}=6-7$ mice per genotype and diet. (B) Weight curve of mice in (A). (C) Insulin tolerance test post 26 weeks ND, $n=3-4$ mice per genotype. (D) Insulin tolerance test of 1 year old WT and $T r e m 2^{-/-}$mice fed a ND, $n=4-5$ mice per genotype. Results are mean \pm SEM. Statistical analysis was performed with two-way ANOVA followed by Bonferroni post-test. * $\mathrm{P}<0.05$, ** $\mathrm{P}<0.01, * * * \mathrm{P}<0.001$, **** $\mathrm{P}<0.0001$. 
A
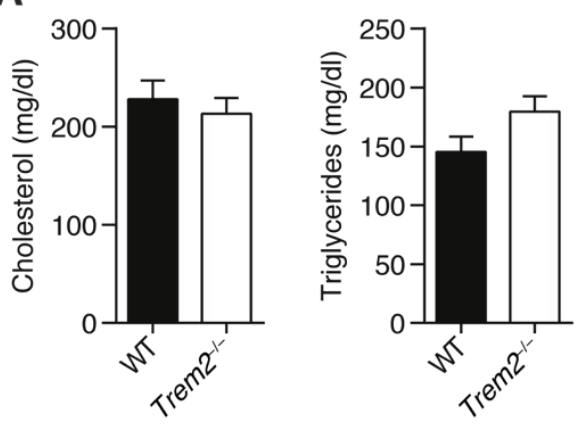

C

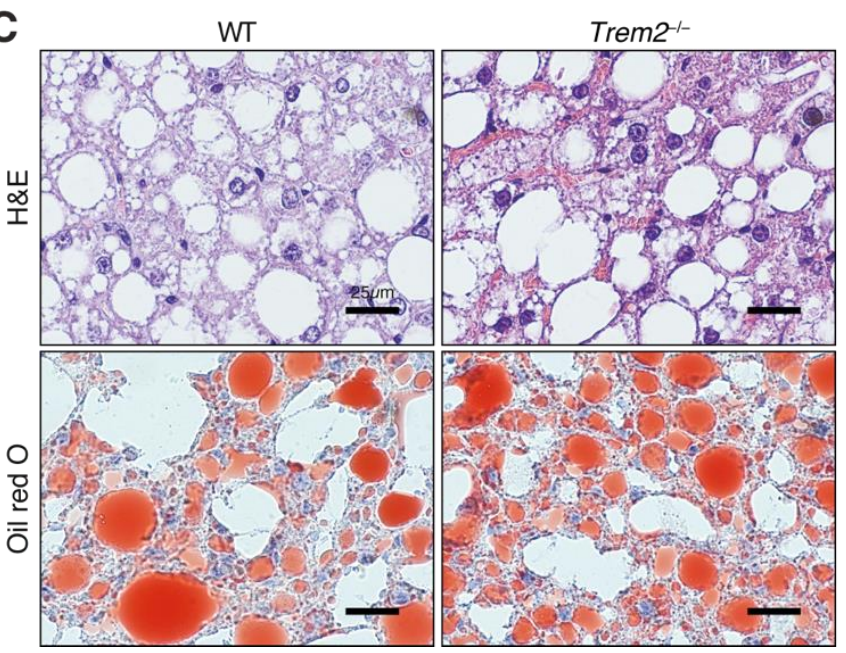

B

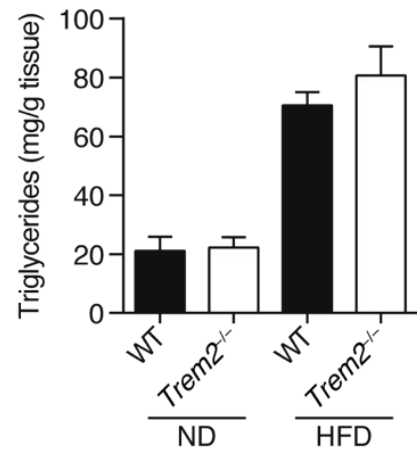

D

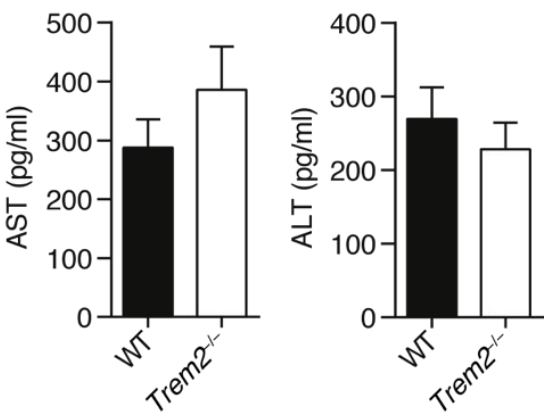

Supplemental Figure. 2 Liver and serum parameters 13 weeks post HFD. (A) Serum cholesterol and triglyceride levels of mice fed a HFD for 13 weeks, $n=7-9$ mice per genotype. (B) Liver triglyceride levels of both genotypes of mice fed a normal chow diet (ND) or HFD for 13 weeks, $n=4$ mice per genotype, ND and 7 mice per genotype HFD. (C) Representative liver sections (H\&E, oil Red O), 13 weeks post HFD. (D) Serum levels of liver enzymes 13 weeks post HFD, $n=7-9$ mice per genotype. Results are mean \pm SEM and are pooled data from 2 independent experiments. 
A

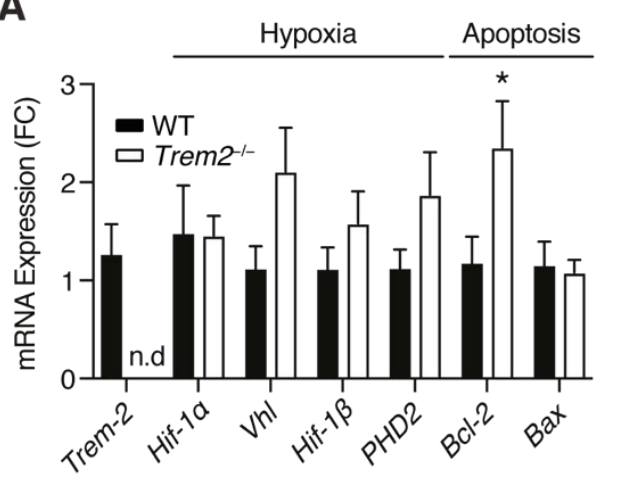

B
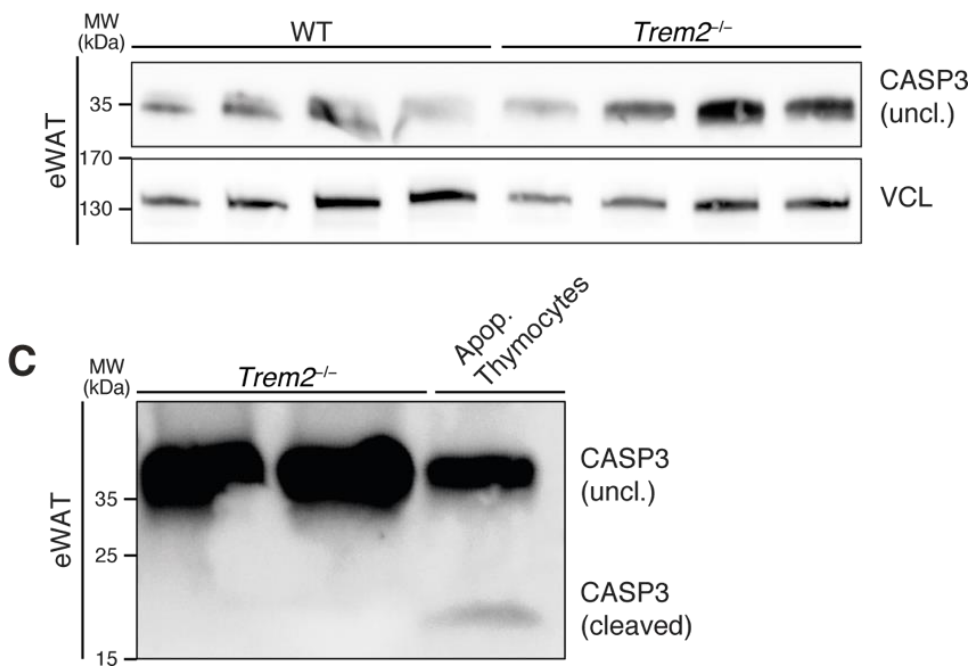

Supplemental Figure. 3 Hypoxia or apoptosis related gene expression and caspase 3 expression in epididymal white adipose 13 weeks post HFD. (A) Hypoxia and apoptosis related gene expression in epididymal white adipose (eWAT) of both genotypes fed at HFD for 13 weeks, $\mathrm{n}=8$ mice per genotype. (B) Caspase 3 western blot of eWAT of both genotypes fed a HFD for 13 weeks, $n=4$ mice per genotype (C) Confirmation the caspase 3 antibody detects cleaved caspase 3 in murine apoptotic thymocytes but not in eWAT. Depicted in this blot is eWAT from two 13 week HFD fed Trem $^{-/-}$animals. Results are mean \pm SEM and in (A) are pooled from 2 independent experiments. Statistical analysis was performed with a Students t-test. $* \mathrm{P}<0.05$ 
A
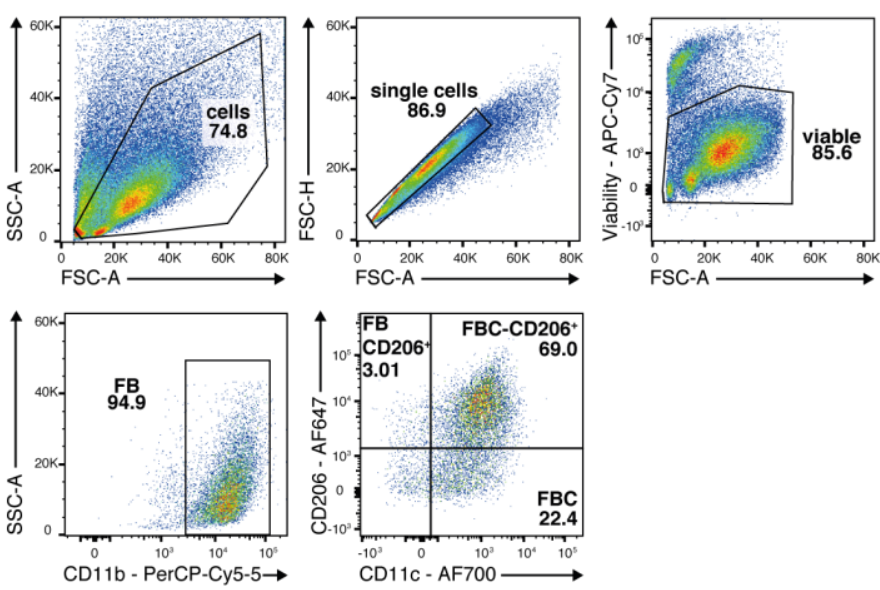

B
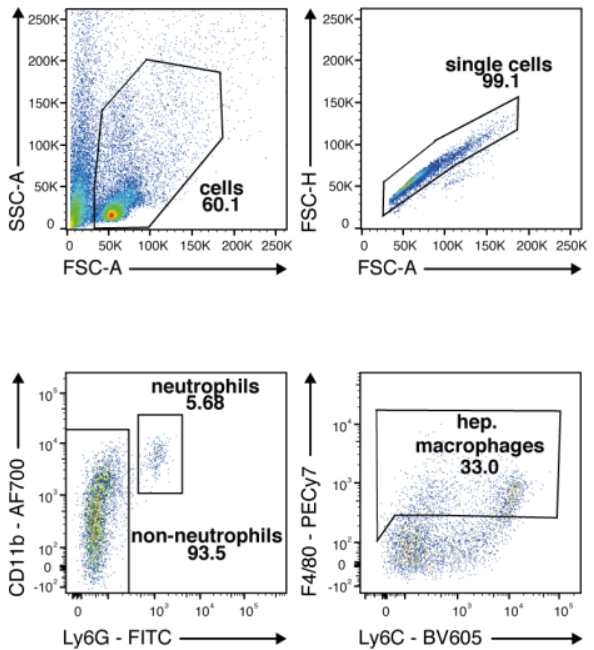

D
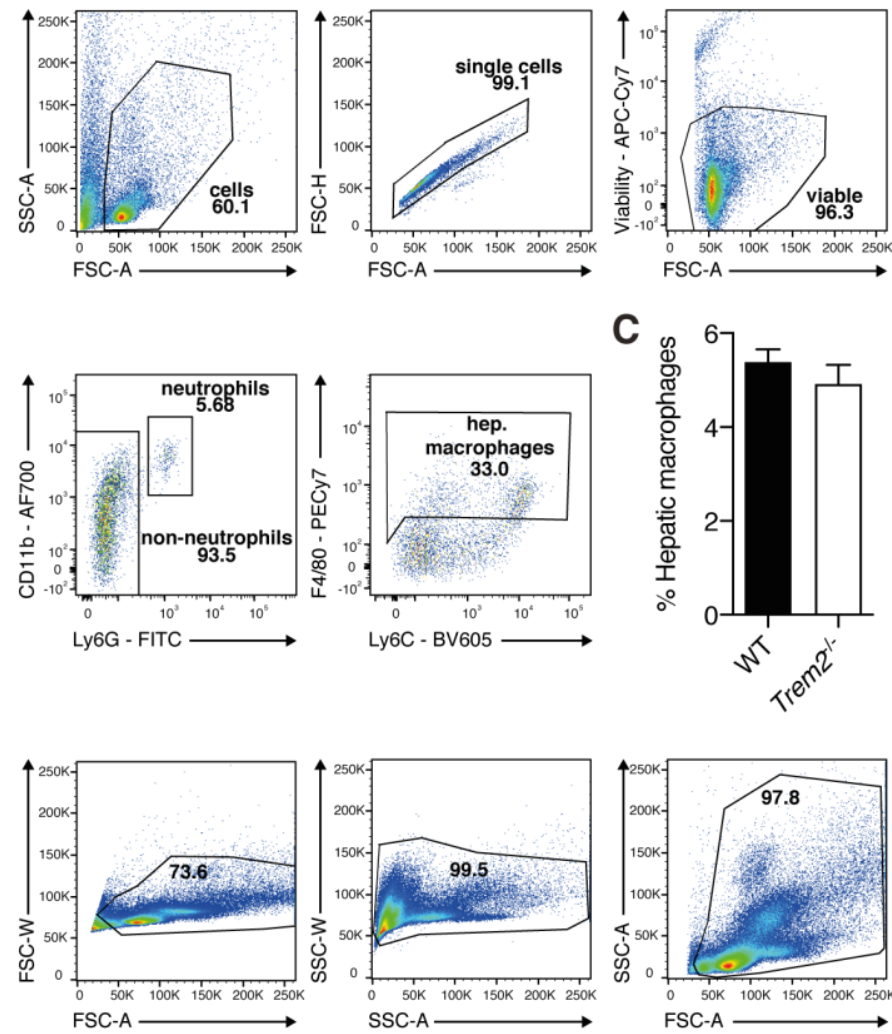
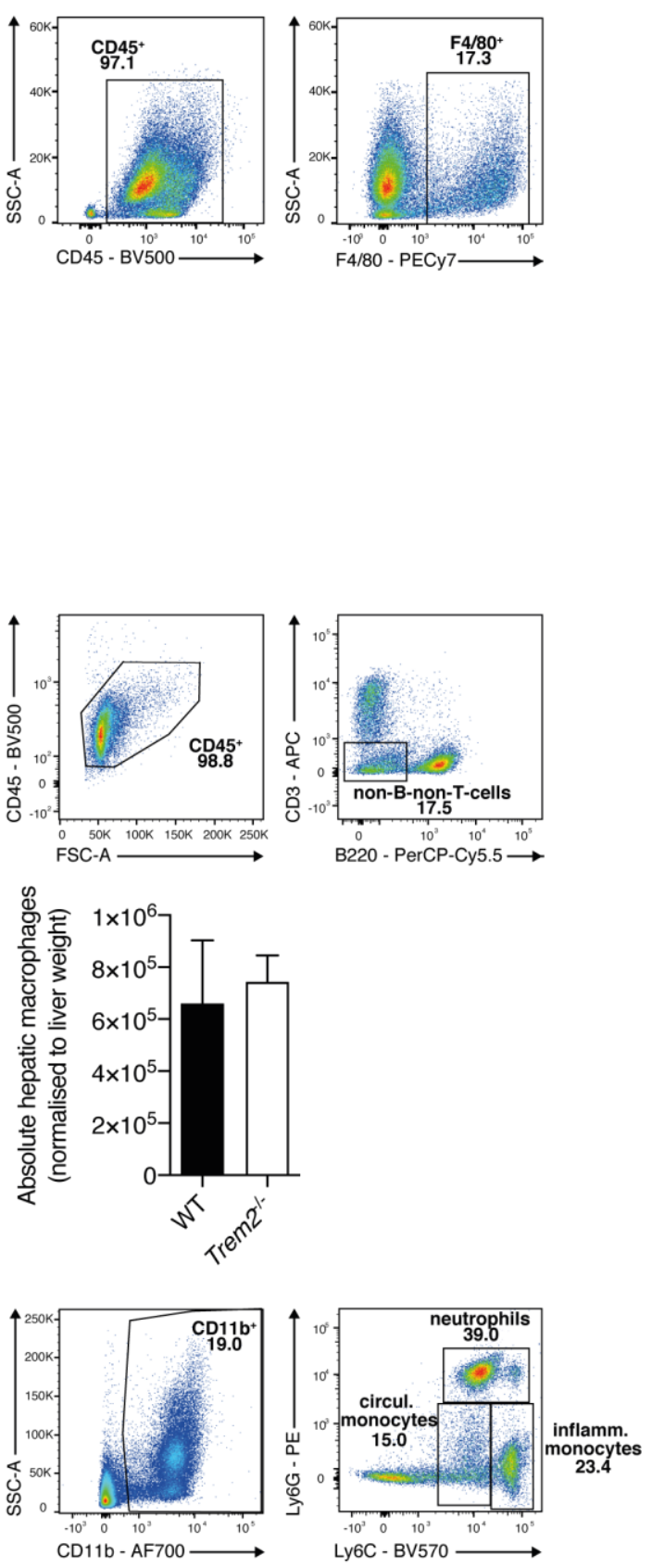

E

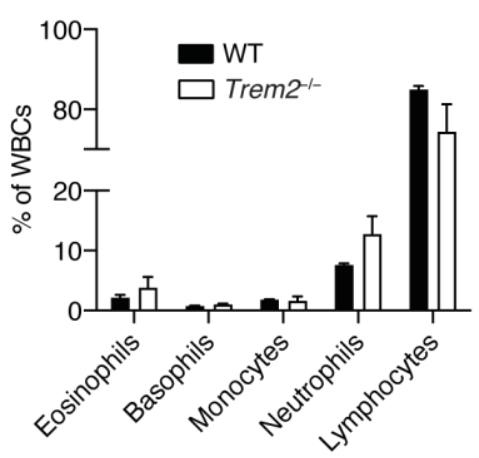


Supplemental Figure. 4 Gating strategy for adipose tissue macrophages (ATMs), hepatic macrophages and white blood cells (WBCs). Hepatic macrophage \% and absolute number and white blood cell counts post 26 weeks HFD in both genotypes. (A) ATMs were defined as viable $\mathrm{CD} 45^{+} \mathrm{F} 4 / 80^{+} \mathrm{CD} 11 \mathrm{~b}^{+}$cells and were further sub-gated for CD11c and CD206. (B) Hepatic macrophage gating strategy. Tissue resident liver macrophages were defined as viable $\mathrm{CD}^{4} 5^{+} \mathrm{CD} 3^{-}$

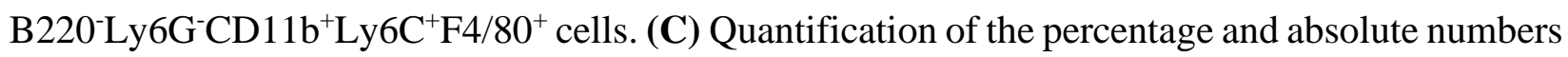
of liver F4/80 ${ }^{+}$cells gated as in (B) in WT and Trem $^{-/-}$mice fed a HFD for 26 weeks, $\mathrm{n}=3$ mice per genotype. (D) Gating strategy for blood monocytes and neutrophils. Neutrophils were defined

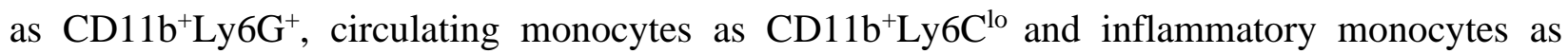
CD11b $^{+}$Ly6C $^{\text {hi }}$. (E) White blood cells counts of obese WT and Trem2 ${ }^{-/-}$mice fed a HFD for 26 weeks, $\mathrm{n}=3-4$ mice per group. 
A

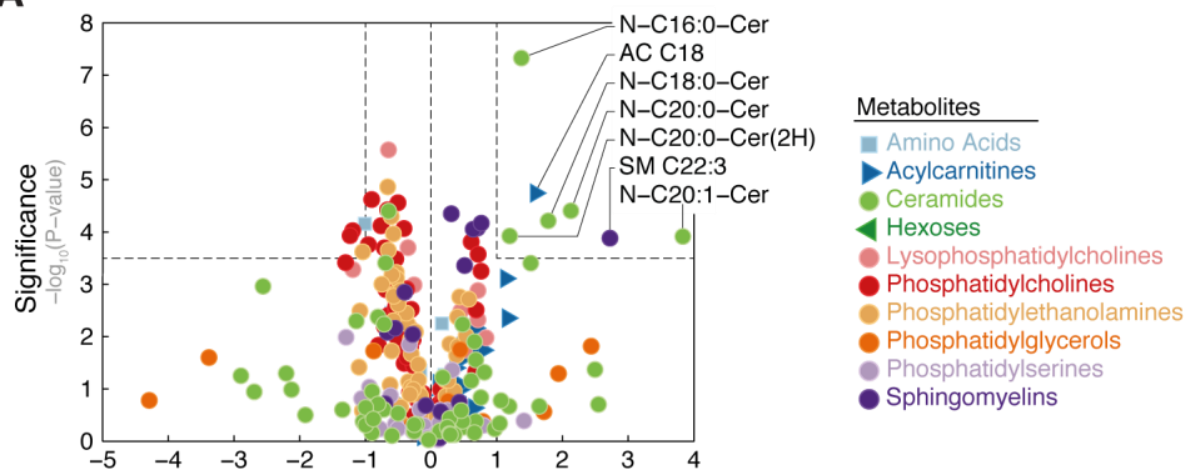

ITT (45') high vs. low responder metabolite difference $\log _{2}$ (fold change in lipid abundance)

B
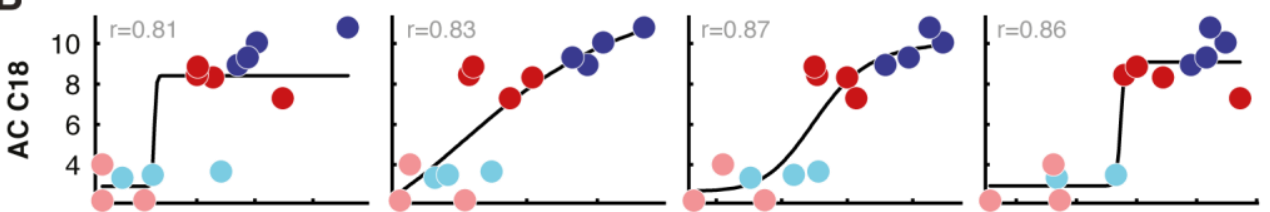

OWT ND

OWT HFD
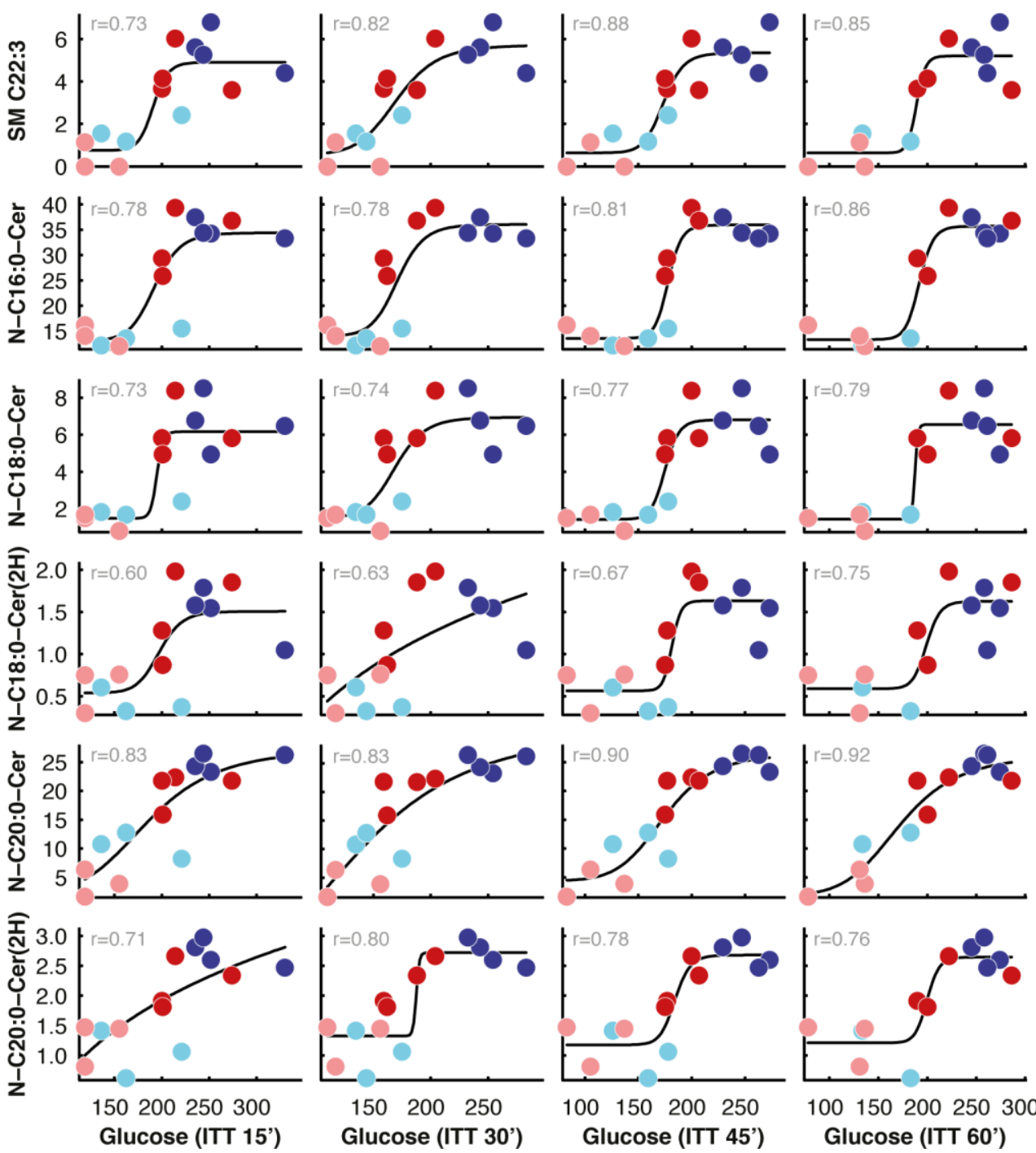

100150200250

100150200250300

Glucose (ITT 45')

Glucose (ITT 60') 


\section{Supplemental Figure. 5 Obesity triggered synthesis of long chain ceramides is associated with}

hyperglycemia upon insulin treatment. (A) $\log _{2}(\mathrm{FC})$ metabolite levels in the 5 mice with the highest glucose levels after $45 \mathrm{~min}$ of insulin treatment compared to the 5 mice with the lowest levels (x-axis) plotted against the significance of the corresponding metabolite difference $\left(\log _{10}(\mathrm{P}-\right.$ value); y-axis). Dots represent metabolites, colored as indicated in legend. (B) Glucose levels (xaxis) versus relative abundance of selected lipids (y-axis) following 15, 30, 45 and 60 min of insulin challenge. Dots represent mice, colored as indicated in legend. 

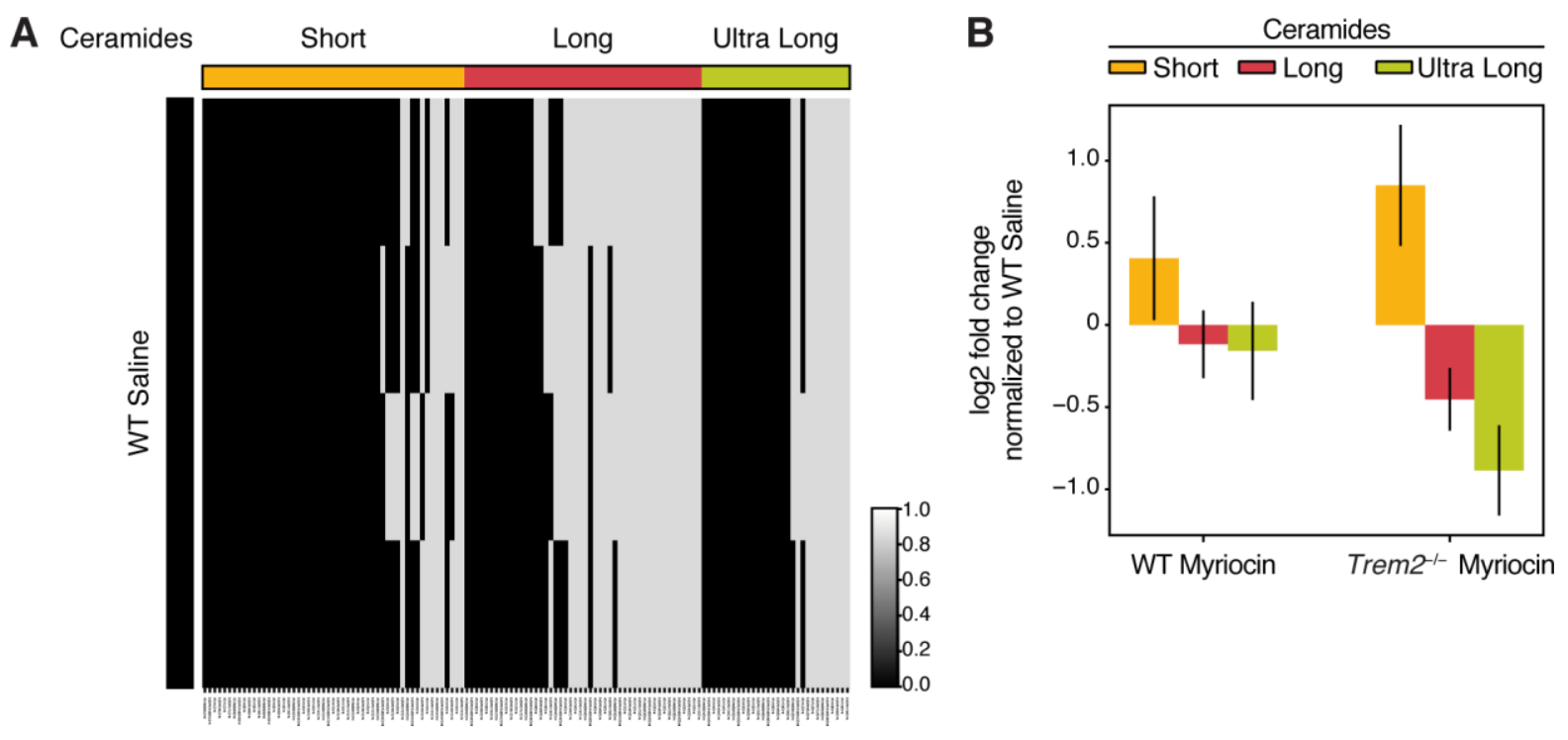

Supplemental Figure. 6. Reproducible upregulation of long chain ceramides 26 weeks post HFD and impact of myriocin on ceramide synthesis in both genotypes. (A) Serum levels of short $(\mathrm{C}<16)$, long $(\mathrm{C} 16-\mathrm{C} 24)$ and ultra-long (> C24) ceramides in 4 WT animals 26 weeks post HFD feeding. Long chain ceramides are especially prevalent. Black indicates ceramides below the detection limit and grey indicates those detected regardless of concentration. Short, long and ultralong ceramides are depicted in yellow, red and green respectively. (B) Bar charts represent the average Log2-fold change (FC) in abundance of short, long and ultra-long chained ceramides for both genotypes of myriocin treated animals compared to WT saline controls. $\mathrm{n}=4$ mice per condition. Results in (B) are mean \pm SEM. 

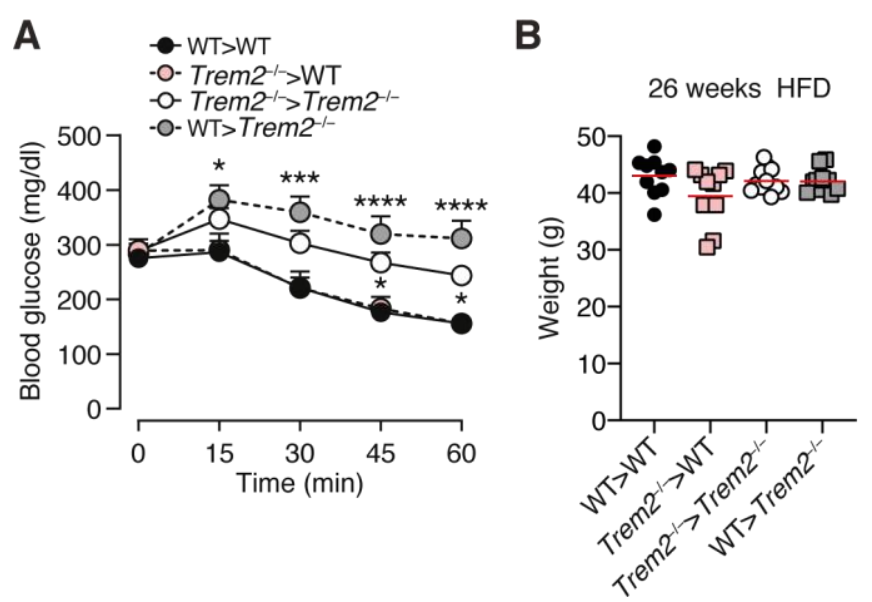

C
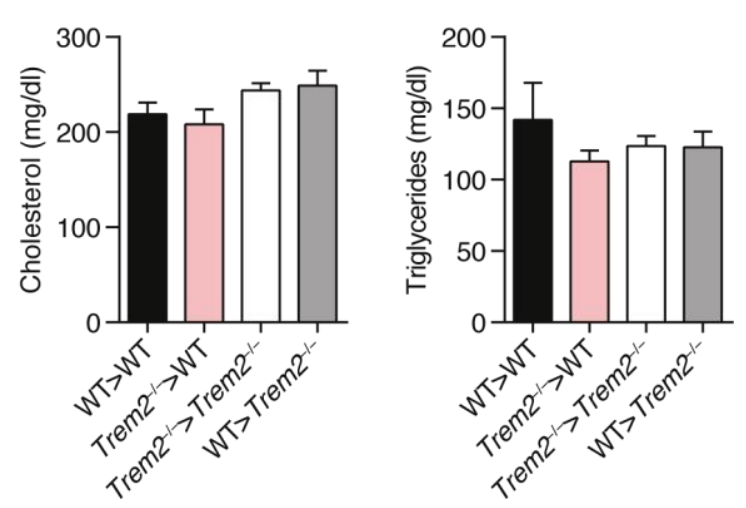

Supplemental Figure. 7 Secondary liver steatosis of $\operatorname{Trem}-2^{-/-}$mice is independent of weight,

triglyceride and cholesterol levels. (A) Insulin tolerance test of bone marrow transplanted mice

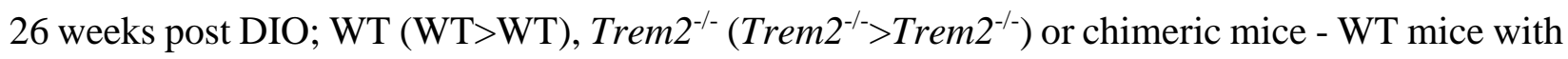
Trem $2^{---}$bone marrow $\left(\right.$Trem $2^{--}>$WT $)$and Trem $^{---}$transplanted with WT bone marrow $\left(\mathrm{WT}>\operatorname{Trem}^{-/}\right)$. (B) Weights of mice in (A) and Figure. 6D-H. (C) Serum cholesterol and triglyceride levels of mice in (A-B) and Figure. 6D-H. Data represent mean \pm SEM and are pooled data from 2 independent experiments. Statistical analysis was performed with two-way ANOVA followed by Bonferroni post-test (A). $\mathrm{n}=10-12$ mice per genotype. $* \mathrm{P}<0.05, * * * * \mathrm{P}<0.0001$ and are versus WT $>$ WT group. 
A

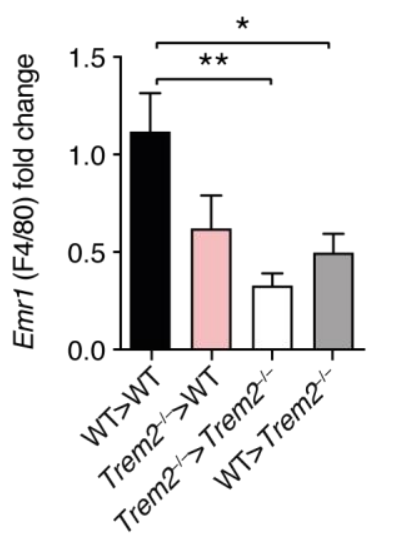

B

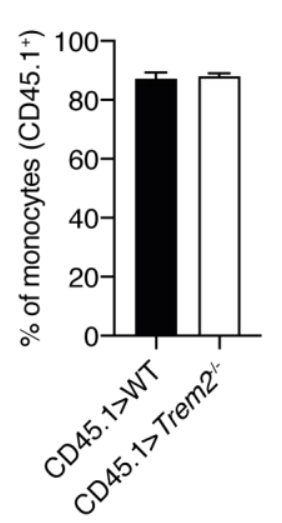

D

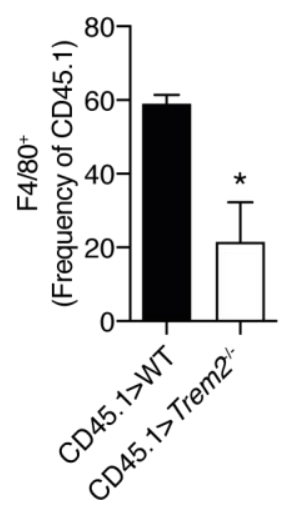

C

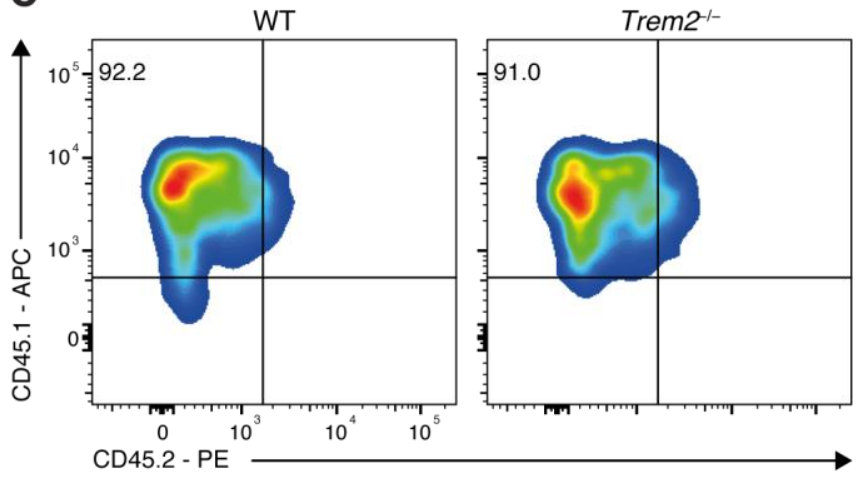

\section{Supplemental Figure. 8 BMT confirms that signals derived from TREM2 deficient adipose}

regulate ATM content. (A) F4/80 transcript levels in bone marrow transplanted mice 26 weeks post DIO; WT $(\mathrm{WT}>\mathrm{WT}), \operatorname{Trem}_{2} 2^{--}\left(\operatorname{Trem} 2^{-/-}>\operatorname{Trem} 2^{-/-}\right)$or chimeric mice - WT mice with Trem2 $2^{-/-}$ bone marrow $\left(\right.$ Trem $\left.2^{--}>\mathrm{WT}\right)$ and $\operatorname{Trem} 2^{---}$transplanted with WT bone marrow $\left(\mathrm{WT}>\operatorname{Tr} e m 2^{--}\right)$. $(\mathbf{B})$ $\%$ of CD45.1 monocytes (defined as CD11b ${ }^{+}$Ly6G-F4/80-CD45.1 $1^{+}$) in CD45.2 WT and Trem2 $^{-/-}$ mice following bone marrow transplantation (BMT) with CD45.1 bone marrow and HFD feeding for 26 weeks, $n=4$ mice per group. (C) Representative FACS plot of (B). (D) Frequency of CD45.1 macrophages (defined as viable, $\mathrm{CD} 45.1^{+} \mathrm{F} 4 / 80^{+}$) in epididymal white adipose (eWAT) post BMT of CD45.2 WT and Trem2 $^{--}$animals transplanted with CD45.1 bone marrow and subsequently fed a HFD for 26 weeks, $\mathrm{n}=4$ mice per group. 


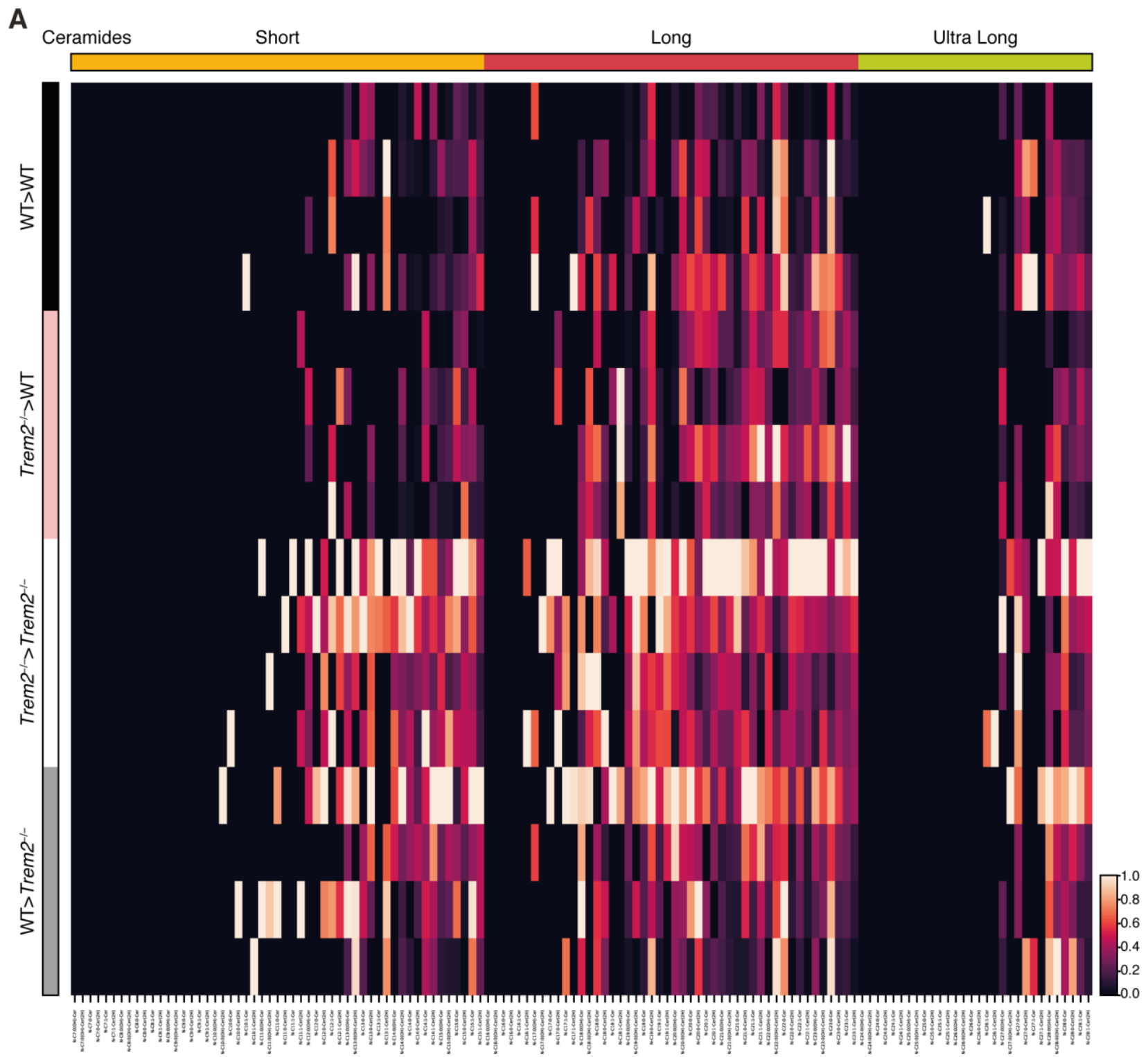

Supplemental Fig. 9 Adipose ceramides of individual animals in Fig. 8D Abundance of short, long and ultra-long ceramide species in epididymal white adipose of bone marrow transplanted mice 26 weeks post HFD. $n=4$ mice per genotype. Overall, higher abundance of long chain ceramides in TREM2 recipient mice. Ceramides are ordered by group from left to right by average highest abundance and individual ceramide abundances scaled between 0 (minimum) and 1 (maximum). 
Supplemental Table 1. Primers used in this study.

\begin{tabular}{|c|c|c|}
\hline Gene & Reference Sequence & Primer Sequence \\
\hline \multicolumn{3}{|r|}{ Mouse } \\
\hline Adipoq & NM_009605.4 & $\begin{array}{l}\text { (Forward) AGCATCCTGAGCCCTTTTGGTGT } \\
\text { (Reverse) TAAGCTGGGGTCTGCCTGTCC }\end{array}$ \\
\hline$F 4 / 80$ & NM_010130.4 & $\begin{array}{l}\text { (Forward) AAAGGGAAGGCTTTCTTCATTG } \\
\text { (Reverse) TGACACTCATTCACACCACCA }\end{array}$ \\
\hline Gapdh & NM_008084 & $\begin{array}{l}\text { (Forward) GGTCGTATTGGGCGCCTGGTCACC } \\
\text { (Reverse) CACACCCATGACGAACATGGGGGC }\end{array}$ \\
\hline Hprt & NM_013556 & $\begin{array}{l}\text { (Forward) GTTAAGCAGTACAGCCCCAAAATG } \\
\text { (Reverse) AAATCCAACAAAGTCTGGCCTGTA }\end{array}$ \\
\hline Trem2 & NM_031254 & $\begin{array}{l}\text { (Forward) CTGGCCTGCGTTCTCCTGA } \\
\text { (Reverse) GGTGGAGGAGGGGAGAGCAT }\end{array}$ \\
\hline $\operatorname{Tnf}$ & NM_013693 & $\begin{array}{l}\text { (Forward) CCACCACGCTCTTCTGTCTAC } \\
\text { (Reverse) AGGGTCTGGGCCATAGAACT }\end{array}$ \\
\hline Il6 & NM_031168 & $\begin{array}{l}\text { (Forward) CAAGTCGGAGGCTTAATTACACATG } \\
\text { (Reverse) ATTGCCATTGCACAACTCTTTTCT }\end{array}$ \\
\hline$I l 1 b$ & NM_008361 & $\begin{array}{l}\text { (Forward) GGACAGAATATCAACCAACAAGTGATA } \\
\text { (Reverse) GTGTGCCGTCTTTCATTACACAG }\end{array}$ \\
\hline Nos2 & NM_010927.4 & $\begin{array}{l}\text { (Forward) TGAAGAAAACCCCTTGTGCT } \\
\text { (Reverse) TTCTGTGCTGTCCCAGTGAG }\end{array}$ \\
\hline Hifla & NM_001313920.1 & $\begin{array}{l}\text { (Forward) ACCTTCATCGGAAACTCCAAAG } \\
\text { (Reverse) ACTGTTAGGCTCAGGTGAACT }\end{array}$ \\
\hline$V h l$ & NM_009507.4 & $\begin{array}{l}\text { (Forward) CAGCTACCGAGGTCATCTTTG } \\
\text { (Reverse) CTGTCCATCGACATTGAGGGA }\end{array}$ \\
\hline Hiflb & NM_009709.4 & $\begin{array}{l}\text { (Forward) TGTCATCCTGAAGACCAACAA } \\
\text { (Reverse) AAGGAGCTCGTTCTCATCCA }\end{array}$ \\
\hline
\end{tabular}




\begin{tabular}{|l|l|l|}
\hline Phd2 & NM_053207.2 & $\begin{array}{l}\text { (Forward) GCCCAGTTTGCTGACATTGAAC } \\
\text { (Reverse) CCCTCACACCTTTCTCACCTGTTAG }\end{array}$ \\
\hline Bcl2 & NM_009741.5 & $\begin{array}{l}\text { (Forward) CTCGTCGCTACCGTCGTGACTTCG } \\
\text { (Reverse) CAGATGCCGGTTCAGGTACTCAGTC }\end{array}$ \\
\hline Bax & NM_007527.3 & $\begin{array}{l}\text { (Forward) AAGCTGAGCGAGTGTCTCCGGCG } \\
\text { (Reverse) GCCACAAAGATGGTCACTGTCTGCC }\end{array}$ \\
\hline \multicolumn{2}{|c|}{ Human } \\
\hline Gapdh & NM_002046.5 & $\begin{array}{l}\text { (Forward) CCAAGGTCATCCATGACAAC } \\
\text { (Reverse) TGTCATACCAGGAAATGAGC }\end{array}$ \\
\hline Trem2 & NM_018965.3 & $\begin{array}{l}\text { (Forward) ACGAGATCTTGCACAAGGCA } \\
\text { (Reverse) GGTAGAGACCCGCATCATGG }\end{array}$ \\
\hline
\end{tabular}




\section{Supplementary references}

1. Jais A, Einwallner E, Sharif O, Gossens K, Lu TTH, Soyal SM, et al. Heme Oxygenase-1 Drives Metaflammation and Insulin Resistance in Mouse and Man. Cell. 2014;158(1):2540.

2. Lindroos J, Husa J, Mitterer G, Haschemi A, Rauscher S, Haas R, et al. Human but not mouse adipogenesis is critically dependent on LMO3. Cell Metab. 2013;18(1):62-74.

3. Lumeng CN, Bodzin JL, and Saltiel AR. Obesity induces a phenotypic switch in adipose tissue macrophage polarization. J Clin Invest. 2007;117(1):175-84.

4. Sharif O, Gawish R, Warszawska JM, Martins R, Lakovits K, Hladik A, et al. The triggering receptor expressed on myeloid cells 2 inhibits complement component 1q effector mechanisms and exerts detrimental effects during pneumococcal pneumonia. PLoS Pathog. 2014;10(6):e1004167.

5. Carpenter AE, Jones TR, Lamprecht MR, Clarke C, Kang IH, Friman O, et al. CellProfiler: image analysis software for identifying and quantifying cell phenotypes. Genome Biol. 2006;7(10):R100.

6. Watarai H, Nakagawa R, Omori-Miyake M, Dashtsoodol N, and Taniguchi M. Methods for detection, isolation and culture of mouse and human invariant NKT cells. Nat Protoc. 2008;3(1):70-8.

7. Perugorria MJ, Esparza-Baquer A, Oakley F, Labiano I, Korosec A, Jais A, et al. Nonparenchymal TREM-2 protects the liver from immune-mediated hepatocellular damage. Gut. 2018.

8. Saluzzo S, Gorki AD, Rana BMJ, Martins R, Scanlon S, Starkl P, et al. First-BreathInduced Type 2 Pathways Shape the Lung Immune Environment. Cell Rep. 2017;18(8):1893-905.

9. Koberlin MS, Snijder B, Heinz LX, Baumann CL, Fauster A, Vladimer GI, et al. A Conserved Circular Network of Coregulated Lipids Modulates Innate Immune Responses. Cell. 2015;162(1):170-83.

10. Zukunft S, Prehn C, Rohring C, Moller G, Hrabe de Angelis M, Adamski J, et al. Highthroughput extraction and quantification method for targeted metabolomics in murine tissues. Metabolomics. 2018;14(1):18. 